\title{
Experimental Study of Self-Similarity in the Coalescence Growth Regime
}

\author{
M. Zinke-Allmang, ${ }^{(1)}$ L. C. Feldman, ${ }^{(2)}$ and W. van Saarloos ${ }^{(3)}$ \\ (1)Department of Physics, University of Western Ontario, London, Ontario, Canada N6A $3 K 7$ \\ (2) AT\& T Bell Laboratories, 600 Mountain Avenue, Murray Hill, New Jersey 07974 \\ (3) Instituut-Lorentz, University of Leiden, P.O. Box 9506, 2300 RA Leiden. The Netherlands
}

(Received 22 November 1991)

\begin{abstract}
Phase transformation dynamics is of current interest in terms of the scaling behavior of the growth law and the prediction of self-similar cluster size distributions. We present the first experimental data to test model predictions for coalescence, showing that recent Monte Carlo simulations agree with measurements for Ga on GaAs(001). Detailed aspects of the growth and the relationship to the theoretical assumptions are discussed.
\end{abstract}

PACS numbers: $68.35 . \mathrm{Fx}, 68.35 . \mathrm{Rh}, 82.20 . \mathrm{Mj}$

Phase formation and phase separation processes have received significant theoretical attention recently. Of particular interest is the late-stage scaling of the growth law for the daughter phase and the scaling properties of the size distribution which are often determined by only a few fundamental aspects of the growth mode, e.g., whether the order parameter is conserved or not. However, few experimental studies address these questions although the morphological quality of structures with heterointerfaces usually depends on the control of the growth kinetics. For example, final film structures of GaAs on Si [1] are determined by cluster growth and coalescence.

In this Letter we discuss new data for coalescence of liquid, metallic Ga on GaAs(001), an example of threedimensional clusters growing on a two-dimensional substrate. We show that the cluster size distribution is in surprisingly good agreement with a recent scaling theory, even though detailed aspects of the theoretical assumptions are not fulfilled. The only other quantitative experimental investigation of coalescence on surfaces, i.e., a study of water droplets on glass surfaces by Beysens and Knobler [2], focused on the exponent of the coalescence power law and is thus complementary to this study.

The evolution of a phase separation system is usually divided into a nucleation regime, an early transient regime, when local effects dominate and nucleation ceases, and a late-stage growth regime when an asymptotic behavior is reached and self-similarity can be tested. In the late stage, one of two growth processes may dominate: Ostwald ripening, i.e., growth of larger clusters at the expense of smaller clusters driven by the Gibbs-Thomson effect, and/or coalescence, i.e., growth of clusters into each other upon contact due to cluster mobility or due to the size increase as a result of a steady increase of the cluster phase material.

For surface systems the ripening process can be treated analytically [3] based on the Lifshitz-Slyozov-Wagner model in the limit of small concentrations and employing mass conservation. According to this theory and its extensions $[3,4]$ the cluster size distribution $f(r, t)$ for long times approaches the scaling form $f_{s}$,

$$
f(r, t) \rightarrow f_{s}\left(\frac{r}{r_{c}(t)}\right), \quad r_{c}(t) \propto t^{1 / n} .
$$

Here $r$ is the radius of the cluster, $r_{c}(t)$ is the critical cluster radius, and the exponent $n$ depends on the ratelimiting factors for growth and the dimensionality of the problem $(n=4$ for three-dimensional clusters on a surface if surface diffusion is the rate-limiting factor). This scaling behavior has been found in many materials, including several metals and semiconductors on Si [5-7].

The other dominant process in the formation of the cluster size distribution is coalescence. Different microscopic mechanisms of coalescence may be distinguished: (a) static coalescence occurs for immobile clusters which combine when their perimeter lines grow together, e.g., during continuous deposition, and (b) dynamic coalescence occurs when mobile clusters grow together upon impact even without further deposition. In the presence of coalescence it is again natural to expect a scaling form for the cluster size distribution, but the two mechanisms of coalescence will yield different scaling forms and growth exponents.

Figure 1 shows four cluster size distributions for static coalescence that have been proposed. Venables, Spiller, and Hanbücken [8] [Fig. 1(a)] qualitatively predict a bimodal distribution based on kinetic growth rate equations. Cluster size distributions are also obtained in computer simulations by Family and Meakin [9] for a model of random addition of small droplets onto a surface, with coalescence under mass and shape preservation of the clusters [Fig. 1(d)]. The asymptotic distribution shows a power-law decay for small sizes superimposed on a monodispersed, bell-shaped distribution peaked at the mean cluster size. In particular, for three-dimensional clusters on a two-dimensional substrate, analytical arguments and computer simulations favor a scaling form [4]

$$
N_{s}(t) \propto s^{-5 / 3} f_{s}\left(\frac{s}{S(t)}\right), \quad S(t) \propto t^{3},
$$




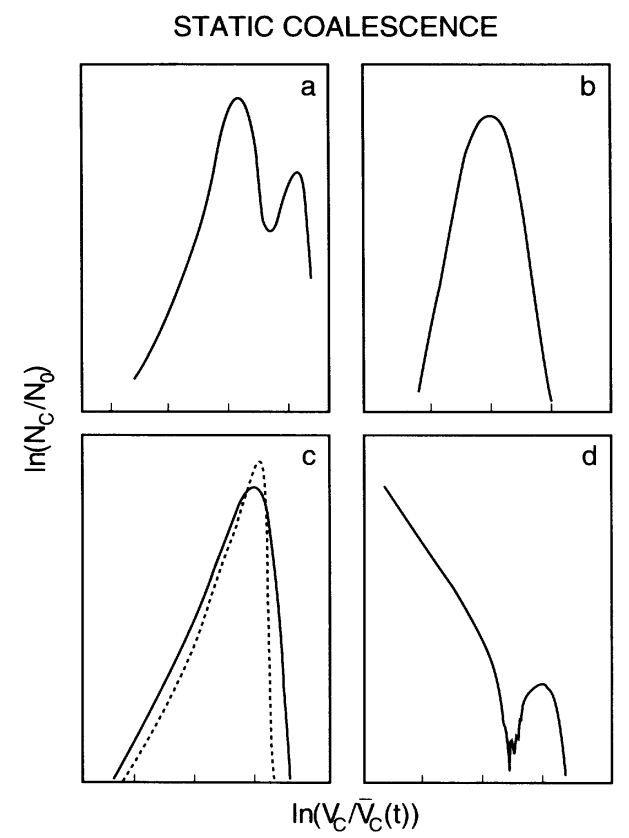

FIG. 1. Theoretical cluster size distributions for static coalescence. Displayed is the ratio of the number of clusters per unit area/volume to $N_{0}=1$ cluster per unit area/volume as a function of the ratio of the cluster volume to the timedependent, mean cluster volume $\bar{V}_{c}(t)$. Predictions are taken from (a) Venables, Spiller, and Hanbücken [8] with the deposition rate $R=$ const, the dimension of space $d_{s}=2$; (b) Vincent [10] with $R \neq$ const, $d_{s}=2$, and the dimension of the cluster $d_{C}=3$; (c) Jayanth and Nash [11] with $R=0, d_{s}=d_{C^{\circ}}=3$; and (d) Family and Meakin [9] with $R=$ const, $d_{s}=2, d_{c}=3$. The dashed line in (c) corresponds to the Ostwald ripening distribution.

where $s$ is the cluster size $\left(s \propto r^{3}\right)$ and $N_{s}(t)$ is the number of clusters of size $s$ at time $t$. Note that this form is more general than Eq. (1), which is only appropriate for cases in which the size distribution is sharply peaked, as in Figs. 1(a)-1(c). Two other distributions, reported by Vincent [10] [Fig. 1(b)] and Jayanth and Nash [11] [Fig. 1 (c)], differ qualitatively in that they predict unimodal distributions. Note that the latter treats a three-dimensional case (cluster solution) and the former is obtained with a variable deposition rate.

We have chosen the system Ga on GaAs(001) for an experimental study of the coalescence regime since (a) previous ion scattering investigations of clustering under mass conservation show that the Ostwald ripening mechanism explains the cluster size evolution in this system, and (b) strikingly simple experiments can reveal a large amount of information on coalescence processes. At high temperatures $\mathrm{Ga}$ is molten, and droplets coalesce when they touch under the action of surface tension.

Sample preparation is done in two different ultrahighvacuum systems (base pressure $<5 \times 10^{-9} \mathrm{~Pa}$ ) equipped with standard surface analytical tools. Samples are mounted on Mo backings with In and are either heated radiatively (Bell Laboratories) or by direct current (University of Western Ontario). Above $\sim 600^{\circ} \mathrm{C}$, temperature control is done by an infrared thermometer; below $600^{\circ}$ we use the heater current which had been previously calibrated via a thermocouple. Clean surfaces are achieved after ex situ chemical oxidation and in situ annealing to $580^{\circ} \mathrm{C}$, and display a $c(2 \times 8)$ low-energy electron diffraction (LEED) pattern [12].

Since the Ga on GaAs(001) ripening studies are partly published elsewhere [6] we only describe those results which are required in the discussion of these new coalescence results. After deposition of about 13 monolayers equivalent coverage of $\mathrm{Ga}$ at room temperature, postdeposit cluster growth was investigated in the temperature regime between 515 and $585^{\circ} \mathrm{C}$ with the cluster height repeatedly measured by ion scattering. The fourth power of the cluster height is found to be linear with time, in agreement with the ripening model of surface diffusion limiting the mass transport [3]. The Arrhenius plot of the growth rate data gives an activation energy for clustering of $\mathrm{Ga}$ on $\mathrm{GaAs}(001)$ of $1.15 \pm 0.20 \mathrm{eV}$, in agreement with the [13] finding of $1.3 \pm 0.1 \mathrm{eV}$ by reflection high-energy electron diffraction [13]. These data reveal absolute growth rates for ripening and allow us to predict the influence of Ostwald ripening at higher temperatures (see Fig. 2).

The coalescence experiments are done at higher temperatures, $T>650^{\circ} \mathrm{C}$, where the amount of metallic $\mathrm{Ga}$ on the surface increases steadily due to continuous arsenic loss (detectable with a mass spectrometer), thus simulating a $\mathrm{Ga}$ deposition experiment. The final structures are investigated by scanning electron microscopy (SEM) and cluster shapes are determined from reflection electron microscopy (REM).

Cluster sizes are obtained by surveying large-area SEM micrographs as shown in Fig. 2(a) for a sample which is heated to a substrate temperature of $660^{\circ} \mathrm{C}$ for $5 \mathrm{~min}$. Varying magnification allows us to analyze a wide range of sizes (from radii of $0.1 \mu \mathrm{m}$ to the largest clusters with $r \sim 15 \mu \mathrm{m})$ with sufficient statistics. Figure 2(b) shows a double-logarithmic plot of the cluster size distribution $N_{s}(t)$ for the same sample as in Fig. 2(a). A total of about 4600 clusters are investigated. The arrow at the lower scale of Fig. 2 indicates the maximum size of clusters if Ostwald ripening would dominate growth, based on previous data [6]. It is obvious that a process other than ripening dominates the structure of this cluster size distribution.

The solid line in Fig. 2(b) shows the cluster size distribution for coalescence-dominated growth, from the Monte Carlo simulation by Family and Meakin [9]. The good agreement between the data and the theoretical curve suggests that coalescence is the dominating cluster growth mechanism. In particular, the bimodal character of the simulation is reproduced experimentally, although 


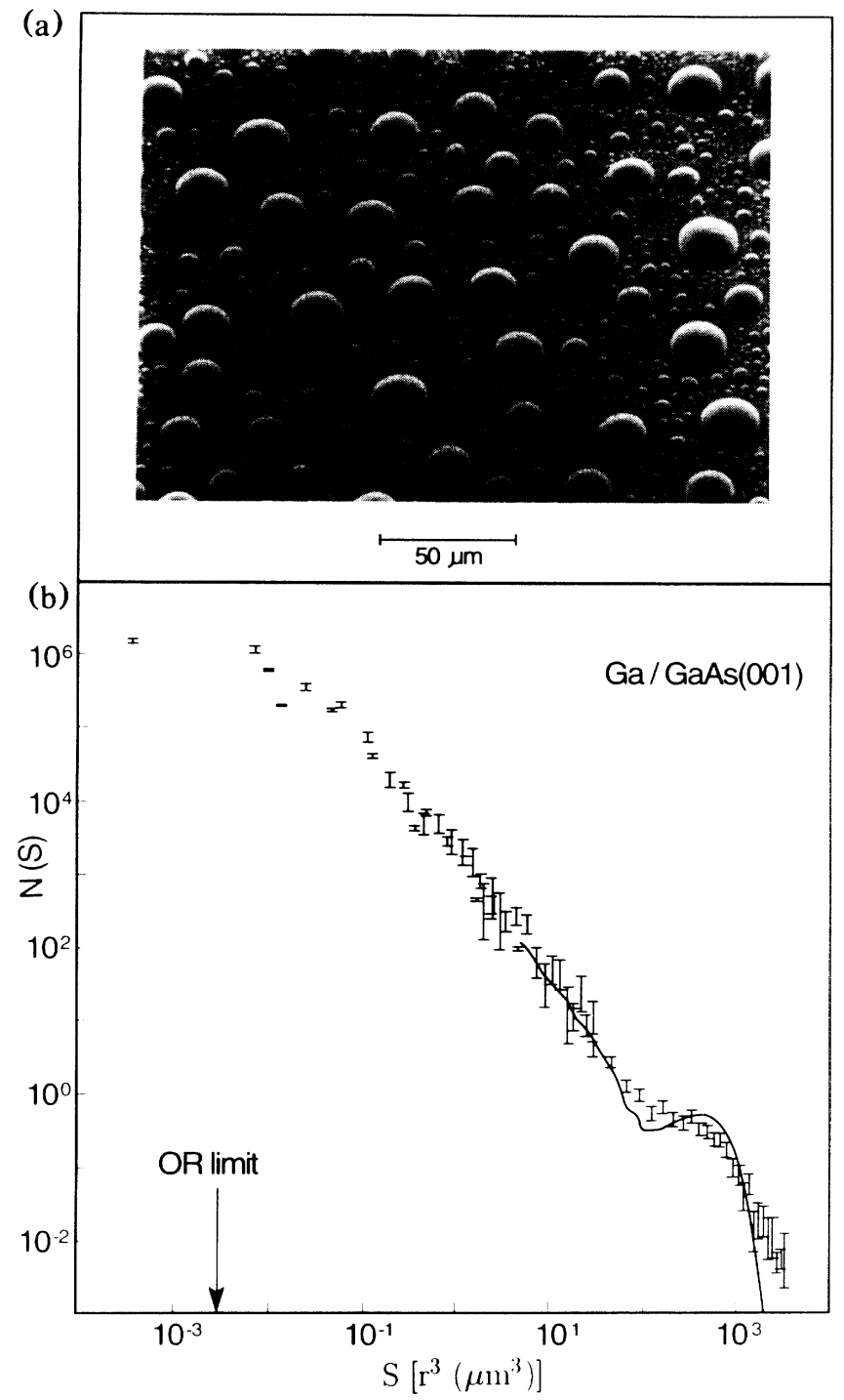

FIG. 2. Experimental cluster size distribution for $\mathrm{Ga}$ on GaAs(00I) after annealing the clean surface to $660^{\circ} \mathrm{C}$ for 5 min. (a) Large-area scanning electron micrograph. (b) Double-logarithmic representation of the corresponding cluster size distribution as a function of cluster size $\left(s \propto r^{3}\right)$. The solid line is a computer simulation by Family and Meakin [9]. The arrow in the lower scale indicates the maximum cluster size when Ostwald ripening would dominate growth.

the constriction between the bell-shaped distribution at larger sizes and the power-law decay of smaller clusters is less distinct in the experiment. Considering the evolution of distributions as shown by Family and Meakin [9], this may indicate that the experimental distribution represents an earlier snapshot than strictly applicable to the theoretical curve. Note in particular that the power-law decay of $N_{s}(t)$ for small $s$ is well reproduced in the experiments. A fit by a power law $s^{-\Theta}$ gives a value of $\Theta$ $=1.6 \pm 0.1$ which agrees well with the predicted value $\Theta$

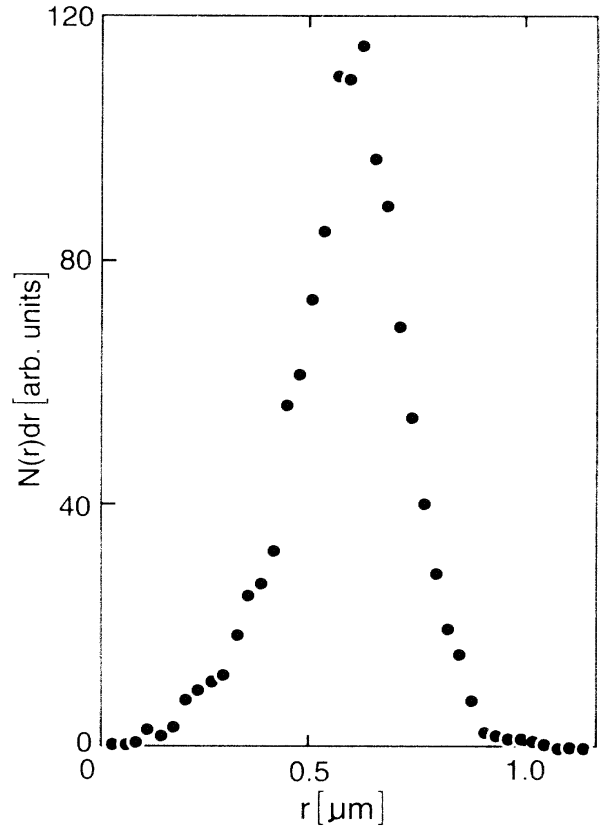

FIG. 3. Experimental Gs cluster size distribution in areas which are cleared in a recent coalescence event.

$=\frac{5}{3}$. Thus we conclude that the cluster size distribution predicted by Family and Meakin describes the distribution expected in systems with coalescence being the dominating growth mechanism. None of the other distributions shown in Fig. 1 fits the data.

This good agreement is somewhat surprising, since the microscopic details of the simulation and our Ga/GaAs experiment differ quite substantially. The Monte Carlo simulation assumes a random addition of small droplets to a surface. Cluster shapes are conserved and no diffusion effects are involved. Experimentally, diffusion effects are clearly observed. This is illustrated in Fig. 3 for the cluster size distribution in those areas that have been cleared in a coalescence event. The different physics in these regions has not been included in the computer simulations.

The first indication of diffusional effects arises from the qualitative observation of denuded zones of uniform width around large clusters in Fig. 2(a). After a large cluster has formed by a coalescence event, its equilibrium adatom concentration is much lower than that of the surrounding small clusters due to the Gibbs-Thomson effect. As a result a reduction in the free adatom concentration occurs in a zone extending with time and limited roughly by the diffusion length. All smaller clusters in this zone dissolve, that is, they donate atoms to the reduced free adatom concentration to achieve equilibrium.

Moreover, Fig. 2(a) shows several examples of areas which were recently cluster free as a result of a coalescence event. They always occur close to a very large cluster. The cluster size distribution in most of these areas is 
qualitatively distinct from the overall cluster size distribution [Fig. 2(b)]. Surveying fifteen such areas with an average diameter of $25 \mu \mathrm{m}$ and a total of 1185 clusters we find the cluster size distribution shown in Fig. 3. This is a linear plot of the probability to find a cluster in a constant radius interval per unit area as a function of radius. Single distributions are rescaled such that the center of mass of all distributions coincide. The average diameter of the clusters, investigated in Fig. 3, is about $0.5 \mu \mathrm{m}$. They display a narrow, unimodal plot, which is characteristically different from coalescencelike distributions such as that shown in Fig. 2(b). These selected areas represent growth systems under exactly the same conditions as the entire system, but with a delayed starting time. Based on the model by Family and Meakin cluster size distributions with a power-law decay with cluster size would be expected. However, only a few of these areas exhibit such indications of coalescence. This suggests that an incubation time precedes coalescence, with other effects, such as diffusion-controlled growth, playing a dominant role.

Another effect not considered in the simulations by Family and Meakin but visible in the experimental study is local ripening [14]. This effect describes competitive effects between two clusters at near proximity based on the Gibbs-Thomson effect but does not include global aspects of the entire distribution. If smaller clusters overlap with the diffusion zone of a larger cluster, the smaller cluster adjusts its curvature locally to that of the larger cluster to accommodate differences in the free adatom concentration between the clusters.

We conclude that the theoretical cluster size distribution by Family and Meakin [9] fits the experimental cluster size distributions in the coalescence regime quite well. The underlying self-similar behavior resulting in this dis- tribution is a strong driving force towards that particular distribution even though microscopic details, such as local ripening or diffusional effects, are substantially different.

We want to thank B. Weir and R. Davidson for assistance. One of us (M.Z.A.) is supported by a generous grant from the National Science and Engineering Research Council of Canada.

[1] D. K. Biegelsen, F. A. Ponce, B. S. Krusor, J. C. Tramontana, and R. D. Yingling, Appl. Phys. Lett. 52, 1779 (1988).

[2] D. Beysens and C. M. Knobler, Phys. Rev. Lett. 57, 1433 (1986).

[3] B. K. Chakraverty, J. Phys. Chem. Solids 28, 2401 (1967).

[4] W. W. Mullins, J. Appl. Phys. 59, 1341 (1986).

[5] L. C. Feldman and M. Zinke-Allmang, J. Vac. Sci. Technol. A 8, 3033 (1990).

[6] M. Zinke-Allmang, Scanning Microsc. 4, 523 (1990).

[7] M. Zinke-Allmang, L. C. Feldman, S. Nakahara, and B. A. Davidson, Phys. Rev. B 39, 7848 (1989).

[8] J. A. Venables, G. D. T. Spiller, and M. Hanbücken, Rep. Prog. Phys. 47, 399 (1984).

[9] F. Family and P. Meakin, Phys. Rev. Lett. 61, 428 (1988).

[10] R. Vincent, Proc. R. Soc. London A 321, 53 (1971).

[11] C. S. Jayanth and P. Nash, J. Mater. Sci. 24, 3041 (1989).

[12] M. D. Pashley, K. W. Haberern, W. Friday, J. M. Woodall, and P. D. Kirchner, Phys. Rev. Lett. 60, 2176 (1988).

[13] J. H. Neave, P. J. Dobson, B. A. Joyce, and Jing Zhang, Appl. Phys. Lett. 47, 101 (1985).

[14] J. P. Hirth, J. Cryst. Growth 17, 63 (1972). 


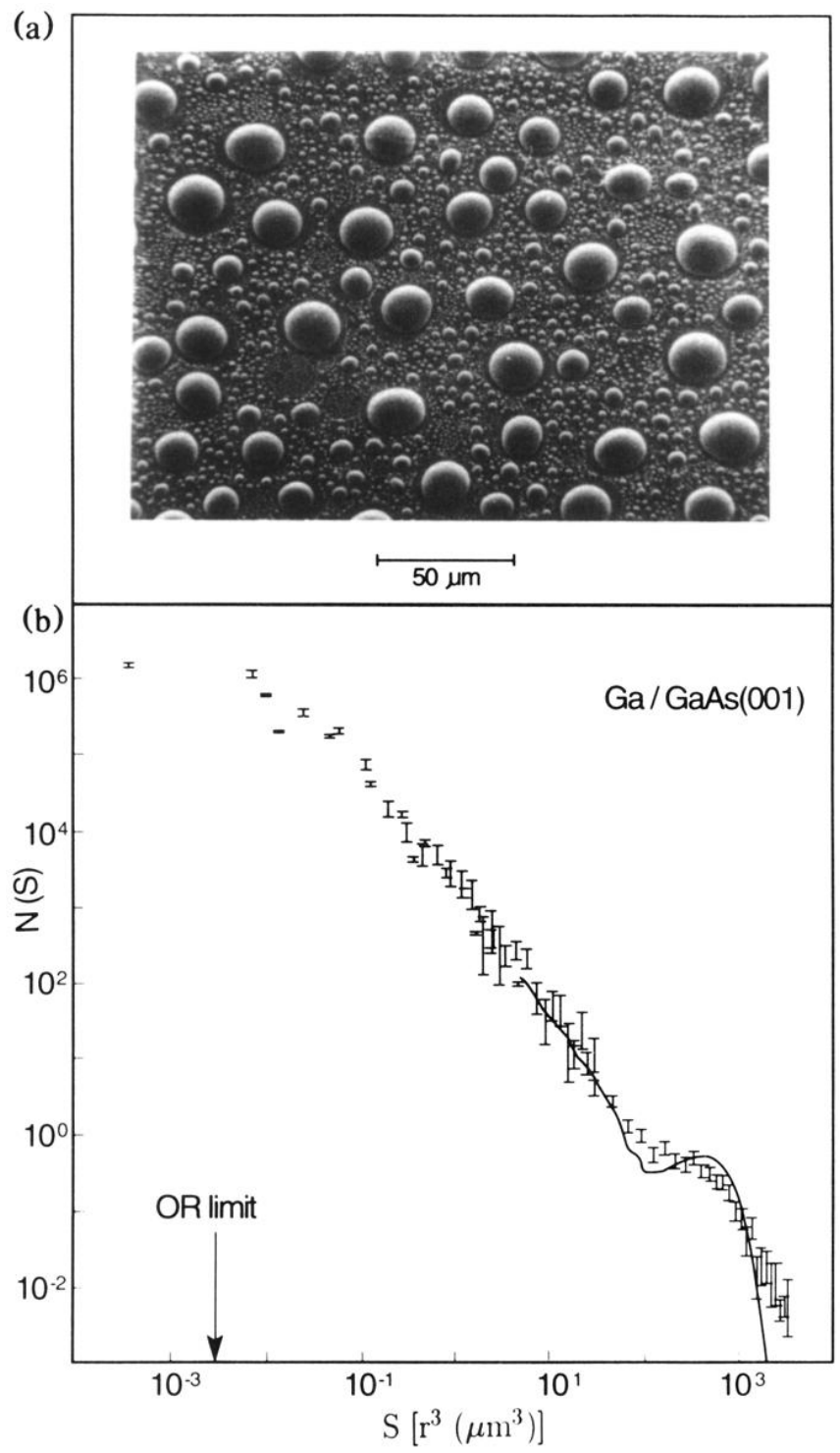

FIG. 2. Experimental cluster size distribution for $\mathrm{Ga}$ on GaAs(001) after annealing the clean surface to $660^{\circ} \mathrm{C}$ for 5 min. (a) Large-area scanning electron micrograph. (b) Double-logarithmic representation of the corresponding cluster size distribution as a function of cluster size $\left(s \propto r^{3}\right)$. The solid line is a computer simulation by Family and Meakin [9]. The arrow in the lower scale indicates the maximum cluster size when Ostwald ripening would dominate growth. 